Developments toward a reliable diode-pumped hydrocarbon-free 795-nm rubidium laser

S. S.Q. Wu, T. F. Soules, R. H. Page, S. C. Mitchell, V. K. Kanz, R. J. Beach

May 8, 2008

CLEO

San Jose, CA, United States

May 4, 2008 through May 9, 2008 
This document was prepared as an account of work sponsored by an agency of the United States government. Neither the United States government nor Lawrence Livermore National Security, LLC, nor any of their employees makes any warranty, expressed or implied, or assumes any legal liability or responsibility for the accuracy, completeness, or usefulness of any information, apparatus, product, or process disclosed, or represents that its use would not infringe privately owned rights. Reference herein to any specific commercial product, process, or service by trade name, trademark, manufacturer, or otherwise does not necessarily constitute or imply its endorsement, recommendation, or favoring by the United States government or Lawrence Livermore National Security, LLC. The views and opinions of authors expressed herein do not necessarily state or reflect those of the United States government or Lawrence Livermore National Security, LLC, and shall not be used for advertising or product endorsement purposes. 


\title{
Developments toward a reliable diode-pumped hydrocarbon-free 795-nm rubidium laser
}

\author{
Sheldon S. Q. Wu ${ }^{1,2, *}$ Thomas F. Soules, ${ }^{1}$ Ralph H. Page, ${ }^{1}$ Scott C. Mitchell, ${ }^{1}$ V. Keith Kanz, ${ }^{1}$ Raymond J. \\ Beach $^{1}$
}

${ }^{1}$ Lawrence Livermore National Laboratory, 7000 East Avenue, Livermore, California 94551, USA

${ }^{2}$ Department of Electrical and Computer Engineering, University of California at San Diego, La Jolla, California 92093-0407, USA

*Corresponding author:wu31@llnl.gov

\begin{abstract}
We report a 795-nm diode-pumpable Rb laser using a buffer gas of pure ${ }^{3} \mathrm{He} .{ }^{3} \mathrm{He}$ gas enhances mixing of the $\mathrm{Rb}$ fine-structure levels. This enables efficient lasing at reduced He pressures and improved thermal management.

OCIS codes: $140.1340,160.3380$.
\end{abstract}

Since the advent of lasers over four decades ago, solid-state and gas lasers have followed largely separate development paths with gas lasers being based primarily on direct electrical discharge pumping or luminescent chemical reactions; and dielectric solid-state lasers being pumped by flash lamps or semiconductor diode laser arrays. In 2002 researchers at Lawrence Livermore National Laboratory demonstrated a new class of laser, combining features from both the gas and solid state laser families, based on diode excitation of atomic alkali vapors [1]. Since that first demonstration of a rubidium resonance transition laser, multiple demonstrations of alkali resonance transition lasers have been reported in the scientific literature using $\mathrm{Rb}$ [2], Cs [3, 4, 5, 6] and $\mathrm{K}$ [7] as the gain media. These systems are pumped on the alkali $D_{2}\left(n^{2} S_{1 / 2} \rightarrow n^{2} P_{3 / 2}\right)$ transition and lased on the $D_{1}$ $\left(n^{2} P_{1 / 2} \rightarrow n^{2} S_{1 / 2}\right)$ transition. Because they are compatible with commercially available laser diode pump sources and show promise for power scaling with good output beam quality and high efficiency, they are being actively investigated today. One common feature of these previous demonstrations has been the use of ethane as the buffer gas or a buffer gas component in the alkali vapor cell. This approach, first suggested by Konefal [8], uses ethane to promote rapid fine-structure mixing, a requirement for efficient laser operation, between the terminal pump level $\left({ }^{2} \mathrm{P}_{3 / 2}\right)$ and the initial laser level $\left({ }^{2} \mathrm{P}_{1 / 2}\right)$. However, due to the ethane in the laser cell this approach has proved problematic from a reliability standpoint due a chemical reaction that takes place between the alkali and ethane resulting in carbonaceous deposits on cell surfaces (e.g. windows) that see high intensity laser light. Following the suggestion of Beach et al [3] who calculated that the $\mathrm{Rb}$-He fine-structure mixing cross section is sufficient to permit efficient diode-pumped $\mathrm{Rb}$ based systems at He buffer gas pressures of $\sim 10$ atm and higher, $\mathrm{Wu}$ et al demonstrated a hydrocarbon-free version of the rubidium laser that obviated the issue of carbon formation and degradation of the vapor cell that we observed in previous alkali laser demonstrations [9].

One potential disadvantage of the pure He buffer gas approach arises from the much smaller Rb F-S mixing cross section of He compared to that of ethane. The impact of these differing cross section values means that to achieve equivalent F-S mixing rates, higher pressures are required in the pure He systems than would be required in systems using ethane as a buffer gas component. However, higher He pressures lead to larger thermal aberrations under equivalent heat loads. The refractive index variation with temperature $\mathrm{dn} / \mathrm{dT}$ in the alkali gain cell is proportional to the He pressure, so higher He pressures will give larger thermal aberrations under equivalent heat loads.

One approach to partially mitigate these issues introduced by the pure He buffer gas is to replace the He buffer gas having a natural isotopic abundance with isotopically enriched ${ }^{3} \mathrm{He}$. The advantage of using isotopically enriched ${ }^{3} \mathrm{He}$ stems from its lower mass and therefore higher thermal velocity at a given temperature in comparison with naturally occurring He. The higher thermal velocity associated with ${ }^{3} \mathrm{He}$ increases the F-S mixing rate, $\gamma_{{ }^{2} P_{32} \rightarrow P_{1 / 2}^{2}}=n_{H e} \sigma_{{ }^{2} P_{3 / 2} \rightarrow P_{1 / 2}^{2}} v_{r}$, where $n_{H e}$ is the number of He atoms per unit volume, $\sigma_{{ }^{2} P_{32} \rightarrow P^{2} P_{12}}$ is the Rb-He F-S mixing cross section, and $v_{r}$ is the mean relative speed between $\mathrm{He}$ and $\mathrm{Rb}$ atoms. First, at a given temperature $v_{r}$ is higher in ${ }^{3} \mathrm{He}$ than ${ }^{4} \mathrm{He}$ by approximately $\sqrt{4 / 3} \approx 1.15$, which not only benefits the F-S mixing rate which depends directly 
on $v_{r}$, but also improves thermal management in the cell. Since the thermal conductivity $\kappa$ of a gas to lowest order is proportional to the mean particle velocity, $\kappa$ of ${ }^{3} \mathrm{He}$ is larger than that of ${ }^{4} \mathrm{He}$ by the same factor. Secondly, the F-S mixing cross section itself has a velocity dependence that is expected to give a $\mathrm{Rb}-{ }^{3} \mathrm{He}$ value larger than the $\mathrm{Rb}-{ }^{4} \mathrm{He}$ value at a given cell temperature due to the difference in thermal speeds of the two He isotopes [10]. Figures 1 and 2 show our experimental setup and data acquired in an initial series of experiments using $2.7 \mathrm{~atm}$ of pure ${ }^{3} \mathrm{He}$ buffer gas and a Ti:sapphire laser as a surrogate for laser diode arrays [11].

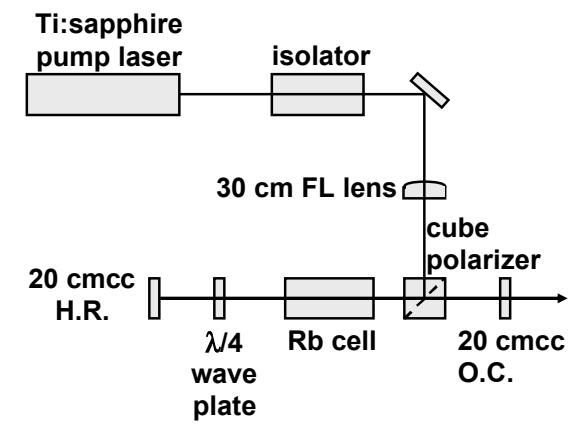

Fig. 1. Schematic diagram of the experimental setup used in our demonstrations.

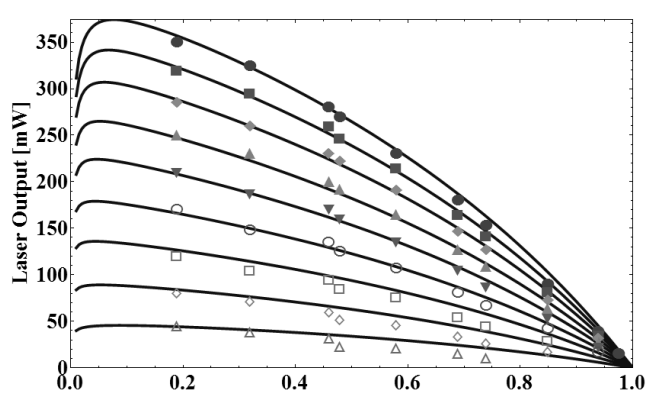

Fig. 2. Rb laser output power for various pump powers plotted against output coupler reflectivity. Solid curves represent model predictions. From top to bottom, pump powers are: 1.65, 1.52, $1.39,1.24,1.1,0.95,0.81,0.66$ and $0.52 \mathrm{~W}$.

The good agreement between measured laser performance (symbols) and our laser model (solid lines) in Fig. 2 supports further pursuit of this approach for power scaling. Obviated is the issue of carbon formation and degradation of the vapor cell that was observed in previous alkali laser demonstrations that used ethane as a buffer gas component. Additionally, we have now demonstrated a similar system using fully laser diode-pumping. The results of this investigation and our power scaling predictions based on our anchored models will be covered.

In view of the advantages of ${ }^{3} \mathrm{He}$ over ${ }^{4} \mathrm{He}$ based systems, we expect the ${ }^{3} \mathrm{He}$ approach to be the preferred route to power scaling DPAL lasers to efficient, reliable, and good beam quality systems. Due to their projected efficiency advantages over diode-pumped solid state lasers (DPSSLs), their compatibility with commercially available laser diode arrays, and now a demonstrated system that promise very high reliability, diode-pumped ${ }^{3} \mathrm{He}-$ only $\mathrm{Rb}$ lasers will potentially compete favorably with DPSSLs in many applications that require high beam quality $\mathrm{cw}$ or quasi-cw laser operation.

This work performed under the auspices of the U.S. Department of Energy by Lawrence Livermore National Laboratory under Contract DE-AC52-07NA27344.

\section{References:}

[1] W.F. Krupke, R.J. Beach, V.K. Kanz, and S.A.Payne, "Resonance transition 795-nm rubidium laser," Optics Letters Vol. 28 , No. 23 pp. 2336-2338 (2003)

[2] R. H. Page, R. J. Beach, V. K. Kanz, and W. F. Krupke, "Multimode-diode-pumped gas (alkali-vapor) laser," Opt. Lett. 31, 353-355 (2006)

[3] R. J. Beach, W. F. Krupke, V. K. Kanz, S. A. Payne, M. A. Dubinskii, and L. O. Merkle, "End-pumped continuous-wave alkali vapor lasers: experiment, model, and power scaling," J. Opt. Soc. Am. B 21, 2151-2163 (2004).

[4] T. Ehrenreich, B. Zhdanov, T. Takekoshi, S. P. Phipps, and R. J. Knize, ”Diode Pumped Cesium Laser”, Electronics Lett. 41, 47-48 (2005).

[5] Y. Wang, T. Kasamatsu, Y. Zheng, H. Miyajima, H. Fukuoka, S. Matsuoka, M.Niigaki, H. Kubomura, T. Hiruma, H. Kan, "Cesium vapor laser pumped by a volume-Bragg-grating coupled quasi-continuous-wave laser-diode array", Appl. Phys. Lett. 88, 141112 (2006).

[6] Boris Zhdanov1, R. J. Knize1, "Diode-pumped $10 \mathrm{~W}$ continuous wave cesium laser," Opt. Lett. 32, 2167-2169 (2007).

[7] B. Zhdanov, C. Maes, T. Ehrenreich, A. Havko, N. Koval, T. Meeker, B. Worker, B. Flusche and R. J. Knize, "Optically Pumped Potassium Laser", Opt. Com. 270, 353-355 (2007).

[8] Z. Konefal, "Observation of collision induced processes in rubidium-ethane vapour", Opt. Com. 164, 95-105 (1999).

[9] S.S.Q. Wu, T.F. Soules, R.H. Page, S.C. Mitchell, V.K. Kanz, and R.J. Beach, "Hydrocarbon-free resonance transition 795-nm rubidium laser," Opt. Lett. 32, 2423-2425 (2007).

[10] A. Gallagher, "Rubidium and Cesium Excitation Transfer in Nearly Adiabatic Collisions with Inert Gases", Phys. Rev. 172, 88 (1968).

[11] S.S.Q. Wu et al., "Resonance transition 795-nm Rubidium laser using ${ }^{3} \mathrm{He}$ buffer gas", Opt. Commun. (2007),

doi:10.1016/j.optcom.2007.10.076 